\title{
Autoimmune Hepatitis Prevalence among Patients from Saudi Arabia with Chronic Liver Disease Referred for Transient Elastography
}

\author{
Rajaa M. Alshanketi, Reem Jabbad, Bayan Baghlaf, Romaysaa Al-Yamani, Majed Alsahafi, \\ Hisham O. Akbar, Hind I. Fallatah
}

Department of medicine, faculty of medicine, King Abdulaziz University, Jeddah, Saudi Arabia

Email: rajaa.alshanketi@gmail.com

How to cite this paper: Alshanketi, R.M., Jabbad, R., Baghlaf, B., Al-Yamani, R., Alsahafi, M., Akbar, H.O. and Fallatah, H.I. (2021) Autoimmune Hepatitis Prevalence among Patients from Saudi Arabia with Chronic Liver Disease Referred for Transient Elastography. International Journal of Clinical Medicine, 12, 251-260.

https://doi.org/10.4236/ijcm.2021.126022

Received: April 7, 2021

Accepted: June 6, 2021

Published: June 9, 2021

\section{Copyright $\odot 2021$ by author(s) and} Scientific Research Publishing Inc. This work is licensed under the Creative Commons Attribution International License (CC BY 4.0).

http://creativecommons.org/licenses/by/4.0/

\begin{abstract}
Background and study aim: Autoimmune hepatitis (AIH) is a chronic liver disease that can lead to progressive liver damage. The prevalence of $\mathrm{AIH}$ among the general population and among chronic liver disease (CLD) patients is variable worldwide. Currently, no published data on the prevalence of AIH among Saudi or among liver disease patients from the region. In this study, we aimed to assess the prevalence of AIH among CLD patients who were referred for transient elastography (FibroScan) in Saudi Arabia. Patients and methods: A retrospective study was conducted among CLD patients who had a transient elastography (FibroScan) during the 3-year study period. We obtained demographic data and test results for serum alanine aminotransferase (ALT), bilirubin, hemoglobin (Hgb), platelets, and international normalized ratio (INR) from all patients. We compared the results of the AIH patients to those of the non-AIH patients with CLD. In addition, for AIH patients, we measured serum immunoglobulin $\mathrm{G}(\operatorname{IgG})$, antinuclear antibody (ANA), smooth muscle antibody (SMA) and ALT at $2-6$ weeks and at $3-4$ months. Results: We included 494 patients, who were predominantly female (271 (60\%)) and Saudi $(299(60.5 \%))$. Thirty patients (6.1\%) had AIH, which represented the $4^{\text {th }}$ most common liver disease. Compared to non-AIH patients, AIH patients were younger (mean ages 49.9 years, SD 14.22 years, and 40.4 years, SD 13.94 years, respectively, $\mathrm{P}=0.001$ ). Patients with AIH had significantly lower Hgb and platelets $(\mathrm{P}=0.008$ for both $)$ and higher ALT, bilirubin and INR $(\mathrm{P}=0.05,0.047$ and 0.019 , respectively). More than $50 \%$ of the AIH patients had cirrhosis. Older age was not associated with advanced disease stage among the AIH patients $(\mathrm{P}<0.001)$. AIH represents the $4^{\text {th }}$ most common cause of CLD in patients referred for transient elastography in Saudi Arabia. AIH tends to be severe in patients at a young age, with a marked biochemical response to steroids and azathioprine treatment.
\end{abstract}




\section{Keywords}

Autoimmune Hepatitis, Chronic Liver Disease, Saudi Arabia, Prevalence, Transient Elastography (FibroScan)

\section{Introduction}

Autoimmune hepatitis (AIH) is chronic inflammatory liver disease with an unknown etiology. AIH can present as either acute or chronic hepatitis along with liver cirrhosis or end-stage liver disease [1] [2] [3] AIH is more common in young and middle-aged females with a female to male ratio of 3 - 4:1 [4] [5] [6]. However, the age at diagnosis varies widely, affecting individuals from childhood to old age [1] [2] [3]. The prevalence of AIH in Western Europe is variable and is estimated at approximately $10-17$ cases/100,000 people [7] [8]. A higher prevalence rate of 42.9/100,000 people was reported from the state [9]. In Asia and New Zealand, the prevalence rate was 24.5 cases/100,000 people, while in contrast, Singapore had a much lower prevalence of 4 cases/100,000 people [10] [11]. The estimated global incidence and prevalence of AIH are 1.7 - 1.9 cases/100,000 people per year and 16.9 - 42.9/100,000 people, respectively [12]. The global prevalence of AIH among individuals with liver disease is not well studied; however, AIH is much less common than viral hepatitis and alcoholic and nonalcoholic fatty liver disease, representing only $4 \%$ of all indications for liver transplantation [12] [13] [14]. Several national reports from Saudi Arabia have addressed the clinical characteristics of $\mathrm{AIH}$; however, the prevalence of $\mathrm{AIH}$ among the general population and among patients from Saudi Arabia with liver disease is not known [3] [4] [5]. The spectrum of clinical presentations of AIH ranges from asymptomatic disease to acute severe liver failure or severe advanced liver cirrhosis [1]-[6]. AIH is characterized by elevated transaminases, liver infiltration with lymphocytes and plasma cells, and positive serum autoantibodies [1] [2] [3]. The standard treatment for AIH is steroids and immunomodulation with azathioprine [1] [2] [3]. In Saudi Arabia, the prevalence of AIH might be underestimated due to the high prevalence of chronic hepatitis $\mathrm{B}$ and $\mathrm{C}$ and the increasing burden of nonalcoholic fatty liver disease (NAFLD) [15] [16]. This study was conducted at King Abdulaziz University Hospital (KAUH), the largest academic medical center in western Saudi Arabia, and aimed to study the prevalence of AIH among patients with chronic liver disease (CLD).

\section{Patients and Method}

Ethical approval was obtained from the research ethics committee of the Faculty of Medicine, King Abdulaziz University.

This is a retrospective study that was conducted at the Gastroenterology/Hepatology Unit at KAUH, Jeddah. The study was conducted over a 3-year period from April 2015 to April 2018. The study population was composed of 
adult patients above 18 years of age with stable CLD who did not show any clinical evidence of decompensation and who had received transient elastography using FibroScan within the study period. We excluded patients who had FibroScan examinations at KAUH but had incomplete data in their files because of parallel follow-ups in other hospitals. Patients who had a superimposed acute liver injury (such as drug-induced injury on top of stable CLD) were also excluded. The data were obtained from the gastroenterology and hepatology unit documents and from patients' electronic files in the hospital information system.

Demographic data were collected, which include age, gender, and nationality (Saudi or non-Saudi). We gathered the following information for all patients: hemoglobin ( $\mathrm{Hgb}$ ), platelet count, serum alanine transaminase (ALT), serum glutamyl transferase (GGT), serum albumin, and serum bilirubin. These results were obtained from tests that were conducted within one month of the FibroScan examination. We also assessed associated comorbidities or autoimmune diseases and evidence of hepatocellular carcinoma, cirrhosis or fatty liver in the imaging examination.

For the AIH patients, we also included the results of the serum immunoglobulin $\mathrm{G}(\mathrm{IgG})$ test that was conducted within 1 month of the FibroScan examination. In addition, we obtained serum ALT levels at $2-6$ weeks posttreatment and at 3 - 4 months posttreatment.

The FibroScan (Echosens; Paris, France; 2005) examination was performed by one expert examiner with more than 5 years of experience in performing the examination. A successful examination was defined as showing at least 10 valid readings with at least a $70 \%$ success rate (interquartile range (IQR) of less than $30 \%)$. The result was read in kilopascals $(\mathrm{kPa})$. Different stages of fibrosis were defined as F0 (less than $6 \mathrm{kPa}), \mathrm{F} 1(6-7.1 \mathrm{kPa})$ for mild fibrosis, F2 (7.2 - 9.4 $\mathrm{kPa})$ for moderate fibrosis, F3 (9.5 - $12.4 \mathrm{kPa})$ for severe fibrosis, and F4 (>12.5 $\mathrm{kPa}$ ) for cirrhosis [17] [18]. The fat content was determined from the captured attenuation parameter (CAP). Radiological examination was performed using an abdominal ultrasound, and fatty liver was defined as fatty infiltration or attenuation of the liver image with or without hepatomegaly. Portal hypertensive features and cirrhosis were defined with an ultrasound examination if there were dilated portal veins, the presence of collaterals or splenomegaly.

The patients were divided into two groups (the AIH group and the non-AIH group), and a comparison of the two groups was performed in terms of clinical features and laboratory results.

CLD was defined as persistently abnormal liver enzymes for $3-6$ months, positive serological markers for viral hepatitis, and/or the presence of laboratory features of portal hypertension (HTN) (such as thrombocytopenia) together with positive serological markers for AIH or viral hepatitis. Nonalcoholic fatty liver disease was diagnosed based on the following criteria: persistently elevated liver enzymes for more than three months, the presence of metabolic syndrome or obesity, a lack of viral and autoimmune markers, and a negative history of hepatotoxic medication use or radiological examination with an ultrasound or 
computerized tomography (CT) abdominal examination.

AIH was defined according to the international group for AIH criteria and the modified criteria for the diagnosis of AIH [19] [20].

Chronic viral hepatitis $B$ and chronic viral hepatitis $C$ were diagnosed based on the presence of a serological viral marker for hepatitis B virus (HBV) or hepatitis $\mathrm{C}$ virus (HCV) and a positive viral load detected by the TaqMan polymerase chain reaction (PCR) method.

Patients who had long lasting Rheumatoid arthritis and were on methotrexate for 10 years or more, they were assessed for methotrexate induced liver fibrosis using fibroscan.

Patients with overlap syndrome were defined on the bases of presence of biochemical and immunological features of two autoimmune liver diseases at the same time. Those include AIH-PBC overlap 2 patients. And AIH-PSC overlap 1 patient.

\section{Statistical Method}

IBM SPSS 22 was used for statistical analysis. Descriptive statistics are described by frequencies, means, and standard deviations. The chi-square test was used to compare the differences in nominal variables between the AIH patients and the patients with other liver diseases. Student's t-test was used to compare the continuous variables between the AIH patients and the patients with other liver diseases. Linear regression analysis was used to study the effect of different variables on the presence of cirrhosis. A P value $\leq 0.05$ was considered significant.

\section{Result}

The total number of patients was 509, and 494 patients were included in the final analysis according to the inclusion criteria. The total number of AIH was 35 and 5 were excluded because of none availability of their laboratory and follow up data on the hospital information system. The majority of the patients were female $(271 ; 60 \%)$, and the majority of patients were Saudi $(299 ; 60.5 \%)$. The mean age was 49.46 years (SD 14.21; range $18-87$ years). The majority of patients were in their forties.

The most commonly identified liver disease among our cohort was chronic hepatitis $\mathrm{B}(\mathrm{CHB})$, followed by chronic hepatitis $\mathrm{C}(\mathrm{CHC})$. Thirty patients with AIH were identified, making AIH the fourth most common liver disease, representing $6.1 \%$ of all patients (Table 1 ). All the patients in our cohort were type I AIH based on the serological markers.

Other baseline characteristics of all patients are shown in Table 2, which compares the characteristics of AIH patients to those of the patients with other liver diseases. In both groups, there were more Saudi patients than non-Saudi patients, and $73 \%$ of AIH patients were Saudi. Sixty percent of patients were females, and forty percent of patients had cirrhosis based on an ultrasound examination. For the continuous variables the data was normally distributed apart from the serum IgG and IgM. There was a significant difference between the 
AIH patients and patients with other liver diseases in terms of age, Hgb, platelet count, serum ALT, serum GGT, serum bilirubin, INR, and CAP (Table 3). The biochemical parameters were normal in $310(66.8 \%)$ of the non-AIH patients. On the other hand, the laboratory test results of the AIH patients varied from a modest elevation in ALT and serum bilirubin to very high levels $(P=0.05$ and 0.047 , respectively) compared to the levels of the non-AIH group (Table 3).

The mean serum IgG value for AIH patients was $24.1 \mathrm{~g} / \mathrm{L}$ (SD 12.44 SE 2.6) (normal up to $16.1 \mathrm{~g} / \mathrm{L}$ ) and the median was $21.4 \mathrm{~g} / \mathrm{L}$. The mean serum IgM for AIH patients was $1.78 \mathrm{~g} / \mathrm{l}$ (SD 1.25 SE 0.26) (normal range $0.5-1.9 \mathrm{~g} / \mathrm{l}$.) and the median was $1.36 \mathrm{~g} / \mathrm{l}$.

Twenty patients had moderately to strongly positive ANA, and 13 patients had moderately to strongly positive SMA.

The distribution of the two groups according to the stage of fibrosis is shown in Table 4 , and there was a significant difference between the AIH and non-AIH patients $(\mathrm{P}<0.001)$.

Table 1. Distribution of patients according to the underlying liver disease.

\begin{tabular}{ccc}
\hline Diagnosis & Number of patients & Percent \\
\hline CHB & 166 & 33.6 \\
CHC & 153 & 31.0 \\
AIH & 30 & 6.1 \\
NAFLD & 111 & 22.5 \\
Methotrexate & 19 & 3.8 \\
Chronic cholestasis of unknown cause & 1 & 0.2 \\
Overlap syndrome & 4 & 0.8 \\
PBC & 3 & 0.6 \\
CHB + CHC & 6 & 1.2 \\
DILI-induced AIH & 1 & 0.2 \\
Total & 494 & 100.0 \\
\hline
\end{tabular}

Table 2. Baseline characteristics of AIH patients compared to those of non-AIH patients.

\begin{tabular}{lcccc}
\hline \multicolumn{2}{c}{ Variable } & AIH, number (\%) & Non-AIH, number (\%) & P value \\
\hline \multirow{2}{*}{ Nationality } & Saudi & $22(73.3 \%)$ & $277(59.7 \%)$ & 0.18 \\
& Non-Saudi & $8(26.7 \%)$ & $187(40.3 \%)$ & \\
Sex & Male & $12(40 \%)$ & $211(45.5 \%)$ & 0.35 \\
Female & $18(60 \%)$ & $253(54.5 \%)$ & 0.001 \\
Age (years) & 40.5 SD 13.940 & 49.9 SD 14.22 & 0.62 \\
Cirrhosis on ultrasound & $12(40 \%)$ & 124 & 0.044 \\
Autoimmune disease & $6(20 \%)$ & $20(4.31 \%)$ & 0.014 \\
\hline
\end{tabular}


Table 3. Baseline laboratory test results of AIH patients compared to those of non-AIH patients.

\begin{tabular}{|c|c|c|c|c|c|}
\hline & Patients & Mean & Std. Deviation & Std. Error Mean & $P$ Value \\
\hline \multirow{2}{*}{$\begin{array}{c}\text { Hgb } \\
\text { Normal } 12-15 \text { g/dL }\end{array}$} & AIH & 12.07 & 2.487 & 0.488 & \multirow{2}{*}{0.008} \\
\hline & Non-AIH & 12.96 & 2.536 & 0.120 & \\
\hline \multirow{2}{*}{$\begin{array}{c}\text { Platelet count } \\
\text { Normal } 150-450 \mathrm{~K} / \mu \mathrm{L}\end{array}$} & $\mathrm{AIH}$ & 216.46 & 117.505 & 23.045 & \multirow{2}{*}{0.008} \\
\hline & Non-AIH & 258.43 & 117.993 & 5.581 & \\
\hline \multirow{2}{*}{$\begin{array}{c}\text { Albumin } \\
\text { Normal } 35-50 \text { g/L }\end{array}$} & $\mathrm{AIH}$ & 31.91 & 12.468 & 2.445 & \multirow{2}{*}{0.245} \\
\hline & Non-AIH & 34.84 & 5.532 & 0.260 & \\
\hline \multirow{2}{*}{$\begin{array}{c}\text { ALT } \\
\text { Normal } 30-65 \mathrm{U} / \mathrm{L}\end{array}$} & $\mathrm{AIH}$ & 83.69 & 79.307 & 15.553 & \multirow{2}{*}{0.05} \\
\hline & Non-AIH & 51.13 & 66.981 & 3.161 & \\
\hline \multirow{2}{*}{$\begin{array}{c}\text { GGT } \\
\text { Normal } 5-85 \mathrm{U} / \mathrm{L}\end{array}$} & $\mathrm{AIH}$ & 237.92 & 265.655 & 52.099 & \multirow{2}{*}{0.004} \\
\hline & Non-AIH & 72.87 & 203.724 & 9.701 & \\
\hline \multirow{2}{*}{$\begin{array}{c}\text { Bilirubin } \\
\text { Normal } 0-17 \mu \mathrm{mol} / \mathrm{L}\end{array}$} & $\mathrm{AIH}$ & 50.13 & 85.957 & 17.191 & \multirow{2}{*}{0.047} \\
\hline & Non-AIH & 14.03 & 25.499 & 1.202 & \\
\hline \multirow{2}{*}{$\begin{array}{c}\text { INR } \\
\text { Normal } 1.1-1.4 \\
\text { Seconds }\end{array}$} & $\mathrm{AIH}$ & 1.35 & 0.474 & 0.106 & \multirow{2}{*}{0.019} \\
\hline & Non-AIH & 1.08 & 0.285 & 0.016 & \\
\hline \multirow{2}{*}{ CAP } & AIH & 217.67 & 64.420 & 15.184 & \multirow{2}{*}{0.033} \\
\hline & Non-AIH & 253.33 & 69.995 & 3.281 & \\
\hline \multirow{2}{*}{ kPa Stiffness score } & $\mathrm{AIH}$ & 13.91 & 9.415 & 1.883 & \multirow{2}{*}{0.10} \\
\hline & Non-AIH & 10.57 & 12.021 & 0.558 & \\
\hline
\end{tabular}

Table 4. The chi square test for the different stage of fibrosis between the two groups.

\begin{tabular}{cccc}
\hline F Score & Number in None AIH & Number in AIH & Total \\
\hline 0 & 191 & 3 & 194 \\
1 & 99 & 5 & 104 \\
2 & 45 & 2 & 47 \\
3 & 33 & 4 & 37 \\
4 & 96 & 16 & 112 \\
& 464 & 30 & 494 \\
\hline
\end{tabular}

Of the AIH patients, 20 patients had available results for follow-up ALT values at 2 - 6 weeks and at $3-4$ months posttreatment with immune suppression. These patients showed a significant reduction in ALT values $(P=0.01$ and 0.004 , respectively).

Of the non-AIH CLD patients, 337 (72.6\%) were older than 40 years, and this age group was associated with a higher stiffness score of $11.72 \mathrm{kPa}$ compared to $7.58 \mathrm{kPa}$ for patients 40 years or younger $(\mathrm{P}>0.001)$.

However, in the AIH group, 11 (36.6\%) patients who were older than 40 years did not have a higher stiffness score than the patients who were 40 years or younger (14.62 $\mathrm{kPa}$ and $13.35 \mathrm{kPa}$, respectively, $\mathrm{P}=0.75)$. 


\section{Discussion}

Our study showed that AIH represents the $4^{\text {th }}$ most common cause of CLD in patients referred for Fibroscan. AIH prevalence among the general population and among patients with CLD is variable in different countries. Some reports have shown that AIH is the cause of $4 \%$ of all indications for liver transplantation [12]. On the other hand, the prevalence within the general population, which ranges from 10.7 - 16.9 cases per 100,000 people in Europe, has been reported [13]. Higher prevalence rates have been reported in some areas such as Alaska, with prevalence rates of 42.9 cases per 100,000 people, and New Zealand, with 24.5 cases per 100,000 people [9] [10]. Similar general population prevalence studies on liver disease have not been conducted in Saudi Arabia. However, the annual Ministry of Health statistics and other reports show a significant burden of $\mathrm{CHB}$ and $\mathrm{CHC}$, which are the two most common liver diseases, as shown in our report [15] [21]. On the other hand, the prevalence of AIH among our cohort is lower than the figure reported by Khalaf et al. [22] and Al Sebayel et al. [23] (14.3\% and 10\%, respectively) among liver transplant patients from Saudi Arabia. This difference can be explained by the severity of AIH among Saudi patients, with more than one-third to more than half of patients having advanced fibrosis or decompensated cirrhosis at a young age [4] [5] [6]. The predominance of AIH hepatitis among females is similar to what has been shown in several national and international studies. On the other hand, we have shown that AIH tends to be a severe disease that has severe fibrosis. This finding is similar to previously published data on AIH from different regions from Saudi Arabia [4] [5] [6]. All patients in our cohort were type I AIH and this is similar to the previously reported data on AIH from our center [5].

The clinical and laboratory features of AIH patients from our cohort are similar to those in the published data on AIH from Saudi Arabia, and they also indicate advanced disease severity compared to the disease severity in non-AIH patients. The disease severity is reflected by lower platelet counts, lower Hgb, and a high rate of patients with advanced fibrosis [4] [5] [6]. On the other hand, the higher serum ALT, GGT and serum bilirubin levels of AIH patients, compared to those of non-AIH patients, may reflect ongoing disease activity in AIH patients in the first set of laboratory testing. The prevalence of severe fibrosis in patients aged older than 40 years was not different from that of younger patients; this lack of difference is another reflection of the prevalence of severe AIH at young ages. This finding is similar to the results discussed in our previous report on AIH patients [5]. AIH is known to respond to steroids and azathioprine in most patients, even those with advanced fibrosis and stable cirrhosis [1] [2] [3], and we have shown a significant biochemical response in serum ALT during a short-term follow-up. However, assessing a longer treatment response was not an aim of our study, and this was previously documented by several national and international studies [1] [2] [3]. Multiple regression analysis showed that among AIH patients, age was associated with evidence of cirrhosis in ultrasound examinations. This finding could reflect a long-term ongoing active disease prior 
to developing cirrhosis at a relatively young age, as shown in most of our AIH patients. This result, in addition to what we have mentioned above, is additional evidence of the aggressive nature of AIH among Saudis [3] [5]. On the other hand, none of the AIH patients had an elevated CAP, which can be explained by the presence of a large number of NAFLD patients representing the most common liver disease after viral hepatitis $\mathrm{B}$ and $\mathrm{C}$. The prevalence of serum autoantibody positivity is similar to what has been shown from previous national data on AIH [3].

Our study may not reflect the real prevalence of AIH and other chronic liver disease from Saudi Arabia, however it gives a reflection on the most common chronic liver disease in Saudi Arabia. In addition, we have shown that AIH though not common but it tends to present with severe form of chronic liver disease. National prevalence studies are lacking about AIH and other liver disease from Saudi Arabia. It is important to have National prevalence studies on AIH since early diagnosis and initiation of immune suppression will delay the progression to advance liver disease in young population. This study might be similar to countries that had high prevalence of viral hepatitis B and C and NAFLD with lower prevalence of AIH and other autoimmune liver disease, like European countries.

In conclusion our study showed that $\mathrm{AIH}$ represents the $4^{\text {th }}$ most common liver disease among patients with liver disease from Saudi Arabia. AIH tends to result in advanced disease in a large percent of patients. Treatment with immunosuppression results in a marked early biochemical response in AIH patients.

\section{Limitations of the Study}

The retrospective nature of the study resulted in failure to include patients who had incomplete data.

The study was conducted in one center, and a similar multicenter national study will more accurately reflect the prevalence of AIH liver disease in the Kingdom of Saudi Arabia.

\section{Funding}

This research did not receive any specific grant from funding agencies in the public, commercial or not-for-profit sector.

\section{Conflicts of Interest}

The authors declare no conflicts of interest regarding the publication of this paper.

\section{References}

[1] European Association for the Study of the Liver (2015) EASL Clinical Practice Guidelines: Autoimmune Hepatitis. Journal of Hepatology, 63, 971-1004.

https://doi.org/10.1016/j.jhep.2015.06.030 
[2] Manns, M.P., Czaja, A.J., Gorham, J.D., Krawitt, E.L., Mieli-Vergani, G., Vergani, D., et al. (2010) Diagnosis and Management of Autoimmune Hepatitis. Hepatology, 51, 2193-2213. https://doi.org/10.1002/hep.23584

[3] Aljumah, A.A., Al Jarallah, B., Albenmousa, A., Al Khathlan, A., Al Zanbagi, A., Al Quaiz, M., et al. (2018) The Saudi Association for the Study of Liver Diseases and Transplantation Clinical Practice Guidelines for Management of Autoimmune Hepatitis. Saudi Journal of Gastroenterology, 24, S1-S20.

https://doi.org/10.4103/sjg.SJG_159_18

[4] Abdo, A.A. (2006) Clinical Presentation, Response to Therapy, and Predictors of Fibrosis in Patients with Autoimmune Hepatitis in Saudi Arabia. Saudi Journal of Gastroenterology, 12, 73-76. https://doi.org/10.4103/1319-3767.27849

[5] Fallatah, H.I., Akbar, H.O. and Qari, Y.A. (2010) Autoimmune Hepatitis: Single-Center Experience of Clinical Presentation, Response to Treatment and Prognosis in Saudi Arabia. Saudi Journal of Gastroenterology, 16, 95-99.

https://doi.org/10.4103/1319-3767.61235

[6] Aljumah, A.A., AlKhormi, A., Al-Ashgar, H., Alsaad, K., Bzeizi, K., Masri, N.A., et al. (2016) Clinical Presentation, Treatment Outcome and Predictors of Severity in Autoimmune Hepatitis: A Retrospective, Multicenter Experience. Journal of Gastroenterology and Hepatology Research, 5, 2147-2151. https://doi.org/10.17554/j.issn.2224-3992.2016.05.663

[7] Invernizzi, P. (2010) Geoepidemiology of Autoimmune Liver Diseases. Journal of Autoimmunity, 34, J300-J306. https://doi.org/10.1016/j.jaut.2009.12.002

[8] Werner, M., Prytz, H., Ohlsson, B., Almer, S., Bjornsson, E., Bergquist, A., et al. (2008) Epidemiology and the Initial Presentation of Autoimmune Hepatitis in Sweden: A Nationwide Study. Scandinavian Journal of Gastroenterology, 43, 1232-1240. https://doi.org/10.1080/00365520802130183

[9] Hurlburt, K.J., McMahon, B.J., Deubner, H., Hsu-Trawinski, B., Williams, J.L. and Kowdley, K.V. (2002) Prevalence of Autoimmune Liver Disease in Alaska Natives. The American Journal of Gastroenterology, 97, 2402-2407. https://doi.org/10.1111/j.1572-0241.2002.06019.x

[10] Ngu, J.H., Bechly, K., Chapman, B.A., Burt, M.J., Barclay, M.L., Gearry, R.B., et al. (2010) Population-Based Epidemiology Study of Autoimmune Hepatitis: A Disease of Older Women? Journal of Gastroenterology and Hepatology, 25, 1681-1686. https://doi.org/10.1111/j.1440-1746.2010.06384.x

[11] Lee, Y.M., Teo, E.K., Ng, T.M., Khor, C. and Fock, K.M. (2001) Autoimmune Hepatitis in Singapore: A Rare Syndrome Affecting Middle-Aged Women. Journal of Gastroenterology and Hepatology, 16, 1384-1389.

https://doi.org/10.1046/j.1440-1746.2001.02646.x

[12] Udompap, P., Kim, D. and Kim, W.R. (2015) Current and Future Burden of Chronic Nonmalignant Liver Disease. Clinical Gastroenterology and Hepatology, 13, 2031-2041. https://doi.org/10.1016/j.cgh.2015.08.015

[13] Blachier, M., Leleu, H., Peck-Radosavljevic, M., Valla, D.C. and Roudot-Thoraval, F. (2013) The Burden of Liver Disease in Europe: A Review of Available Epidemiological Data. Journal of Hepatology, 58, 593-608.

https://doi.org/10.1016/j.jhep.2012.12.005

[14] Wong, M.C.S., Huang, J.L.W., George, J., Huang, J., Leung, C., Eslam, M., et al. (2019) The Changing Epidemiology of Liver Diseases in the Asia-Pacific Region. Nature Reviews Gastroenterology \& Hepatology, 16, 57-73. https://doi.org/10.1038/s41575-018-0055-0 
[15] Memish, Z.A., Knawy, B.A. and El-Saed, A. (2010) Incidence Trends of Viral Hepatitis A, B, and C Seropositivity over Eight Years of Surveillance in Saudi Arabia. International Journal of Infectious Diseases, 14, e115-e120. https://doi.org/10.1016/j.ijid.2009.03.027

[16] Alswat, K., Aljumah, A.A., Sanai, F.M., Abaalkhail, F., Alghamdi, M., Al Hamoudi, W.K., et al. (2018) Nonalcoholic Fatty Liver Disease Burden-Saudi Arabia and United Arab Emirates, 2017-2030. Saudi Journal of Gastroenterology, 24, 211-219. https://doi.org/10.4103/sjg.SJG_122_18

[17] Castera, L., Forns, X. and Alberti, A. (2008) Non-Invasive Evaluation of Liver Fibrosis Using Transient Elastography. Journal of Hepatology, 48, 835-847. https://doi.org/10.1016/j.jhep.2008.02.008

[18] Castera, L., Vergniol, J., Foucher, J., Le Bail, B., Chanteloup, E., Haaser, M., et al. (2005) Prospective Comparison of Transient Elastography, Fibrotest, APRI, and Liver Biopsy for the Assessment of Fibrosis in Chronic Hepatitis C. Gastroenterology, 128, 343-350. https://doi.org/10.1053/j.gastro.2004.11.018

[19] Alvarez, F., Berg, P.A., Bianchi, F.B., Bianchi, L., Burroughs, A.K., Cancado, E.L., Chapman, R.W., Cooksley, W.G., Czaja, A.J., Desmet, V.J., Donaldson, P.T., Eddleston, A.L., Fainboim, L., Heathcote, J., Homberg, J.C., Hoofnagle, J.H., Kakumu, S., Krawitt, E.L., Mackay, I.R., MacSween, R.N., Maddrey, W.C., Manns, M.P., McFarlane, I.G., Buschenfelde, K.H.M.Z., Mieli-Vergani, G., Nakanuma, Y., Nishioka, M., Penner, E., Porta, G., Portmann, B.C., Reed, W.D., Rodes, J., Schalm, S.W., Scheuer, P.J., Schrumpf, E., Seki, T., Toda, G., Tsuji, T., Tygstrup, N., Vergani, D. and Zeniya, M. (1999) International Autoimmune Hepatitis Group Report: Review of Criteria for Diagnosis of Autoimmune Hepatitis. Journal of Hepatology, 31, 929-938. https://doi.org/10.1016/S0168-8278(99)80297-9

[20] Hennes, E.M., Zeniya, M., Czaja, A.J., Pares, A., Dalekos, G.N., Krawitt, E.L., et al. (2008) Simplified Criteria for the Diagnosis of Autoimmune Hepatitis. Hepatology, 48, 169-176. https://doi.org/10.1002/hep.22322

[21] Saudi MOH (2017) Annual Statistic Book. https://www.moh.gov.sa/en/Ministry/Statistics/book/Documents/ANNUAL-STATI STICAL-BOOK-1438H.pdf

[22] Khalaf, H., Mourad, W., El-Sheikh, Y., Abdo, A., Helmy, A., Medhat, Y., et al. (2007) Liver Transplantation for Autoimmune Hepatitis: A Single-Center Experience. Transplantation Proceedings, 39, 1166-1170. https://doi.org/10.1016/j.transproceed.2007.02.030

[23] Al Sebayel, M., Abaalkhail, F., Al Abbad, S., AlBahili, H., Elsiesy, H., Aleid, M., et al. (2017) Liver Transplantation in the Kingdom of Saudi Arabia. Liver Transplantation, 23, 1312-1317. https://doi.org/10.1002/lt.24803

\section{Abbreviations}

AIH: autoimmune hepatitis, CLD: chronic liver disease, ALT: alanine aminotransferase, Hgb: hemoglobin, INR: international normalized ratio, IgG: immunoglobulin G, ANA: antinuclear antibody, SMA:smooth muscle antibody. 